Methods Data on age-standardized incidence and mortality rate up to 2012 and 2015 were retrieved from the Cancer Incidence in Five Continents Volume XI and the WHO mortality database, respectively. The temporal patterns on the incidence of pancreatic cancer in 2003-2012 were assessed for 27 countries. Its mortality changes in 2006-2015 were evaluated for 23 countries. The Average Annual Percent Change (AAPC) of the incidence/mortality trends with a $95 \%$ confidence interval (CI) was estimated using joinpoint regression analysis.

Results The age-standardised incidence ranged between 0.714.2/100,000, with Thailand (AAPC $=4.48,95 \% \mathrm{CI}=2.10-$ 6.91), the Netherlands (AAPC $=2.14,95 \% \mathrm{CI}=0.80-3.50)$ and Australia ( $\mathrm{AAPC}=1.36,95 \% \mathrm{CI}=0.21-2.52$ ) having the highest incidence rise in men. The greatest increase in incidence among women was observed in Malta (AAPC $=6.04$, 95\% $\mathrm{CI}=0.28-12.14), \quad$ the Netherlands $\quad(\mathrm{AAPC}=3.13, \quad 95 \%$ $\mathrm{CI}=2.00-4.28)$ and New Zealand $\quad(\mathrm{AAPC}=2.32, \quad 95 \%$ $\mathrm{CI}=0.61-4.06$ ). (figure 1) In terms of mortality among men, the age-standardised mortality ranged between 2.2-15.7/ 100,000 with Brazil (AAPC $=0.75,95 \% \mathrm{CI}=0.35-1.14$ ), Russia $(\mathrm{AAPC}=0.73,95 \% \mathrm{CI}=0.41-1.05)$ and Spain $(\mathrm{AAPC}=0.56$, $95 \% \mathrm{CI}=0.03-1.10)$ reporting the biggest increase. Spain $(\mathrm{AAPC}=1.53,95 \% \mathrm{CI}=0.96-2.10)$, Japan $(\mathrm{AAPC}=1.41,95 \%$ $\mathrm{CI}=0.85-1.98)$ and Belgium $(\mathrm{AAPC}=1.00$, 95\% $\mathrm{CI}=0.02-$ 1.99) demonstrated the most prominent rise among women. Conclusions Overall, the incidence and mortality rates of pancreatic cancer were still rising in many countries, especially among the female population. In addition to the implementation of regular surveillance and advanced technological management, future research should explore the underlying reasons for these epidemiological trends.

\section{IDDF2019-ABS-0250 EFFECTIVENESS OF PROBIOTICS IN IMPROVING MINIMAL HEPATIC ENCEPHALOPATHY IN PATIENTS WITH COMPENSATED LIVER CIRRHOSIS - A META-ANALYSIS}

Akemi Kimura*. Jose R. Reyes Mem. Medical Center, Philippines

\subsection{6/gutjnl-2019-IDDFabstracts.295}

Background Hepatic encephalopathy (HE) involves a spectrum of potentally reversible neuropsychiatric abnormalities seen in patients with chonic liver disease or cirrhosis. However, clinicians frequently overlook its subclinical features, known as minimal hepatic encephalopathy (MHE). $80 \%$ of these cirrhotic patients are often not given preventive measures. By definition, MHE has no obvious clinical manifestations, often characterized by neurocognitive impairments in psychomotor speed, attention and visual perception. Ammonia is the key toxin in HE, where the highest levels are derived from both the urease- producing bacteria, leadsing to glutamine accumulation in the brain causing overt HE. Probiotics have multiple mechanisms that make them more superior than the conventional therapies, lactulose or poorly absorbed aantibiotics. Since probiotics are safe, natural and well-tolerated, probiotics for MHE may be ideal. They alter the gut mcirobiome which are crucial in the formation of ammonia, inducing an acidic colonic mileau which promotes acidifying bacteria, rather than the urease-producing bacteria. This mechanism prevents MHE from developing into an overt hepatic encephalopathy.
This meta-analysis seeks to determine the efficacy of probiotics in improving MHE and help prevent these cirrhotic patients from developing overt hepatic encephalopathy.

Methods Extensive literature search of 401 published articles from the year 2000-2017 was reviewed. After exclusion, four (4) randomized controlled studies were included in this metaanalysis, which involved 222 patients, 109 patients with MHE were given probiotics while 113 patients were placed in the placebo arm. The main outcome measures were an improvement in MHE and prevention of overt HE. Psychometric tests were used in the diagnosis and monitoring the progress of MHE. Odds ratio (OR) was calculated with 95\% confidence interval (CI) while heterogeneity was assessed using the $\mathrm{I}^{2}$ statistics. Sensitivity testing was two-tailed and set at p-value of 0.05 . The clinical efficacy was determined by the MantelHaenszel procedure for binary date.

Results Improvement in MHE was improved significantly in the treatment group, compared to the placebo arm, was shown in this meta-analysis. (figure 1)

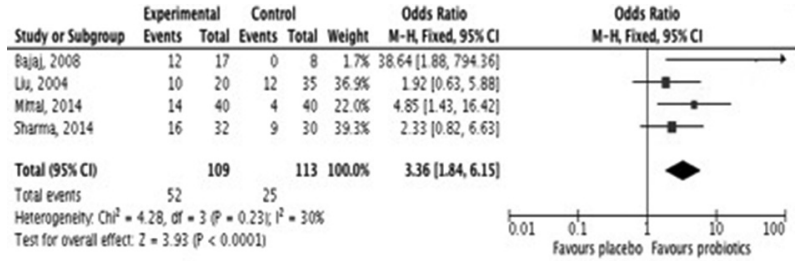

\section{Abstract IDDF2019-ABS-0250 Figure 1}

Conclusions This meta-analysis shows that probiotics may be used to improve MHE and prevent overt HE.

\section{IDDF2019-ABS-0251 THE EFFICACY OF URSODEOXYCHOLIC ACID IN THE TREATMENT OF NON- ALCOHOLIC STEATOHEPATITIS: A 15-YEAR SYSTEMATIC REVIEW}

Higinio Mappala*. Jose R. Reyes Mem. Medical Center, Philippines

\subsection{6/gutjnl-2019-IDDFabstracts.296}

Background Non-alcoholic Fatty Liver (NAFLD) is one of the most common forms of chronic liver disease which may progress to non-alcoholic steatohepatitis (NASH) and eventually develop cirrhosis or liver cancer. Currently, there are no proven therapeutic strategies for such disease. Only healthy lifestyle modification through diet and exercise are proven to afford some benefits. Consequently, most clinical efforts have been directed at treating the components of Metabolic Syndrome. Other pharmacologic interventions are directed at specific pathways potentially involved in the pathogenesis of NAFLD or NASH, e.g., insulin resistance, oxidative stress, pro-inflammatory cytokines, apoptosis, bacterial overgrowth and angiotensin pathways.

However, since the FLINT study, the largest NASH Study to date, no drug ever came close to Obeticholic Acid. (ObA), except Ursodeoxycholic acid (UDCA). This systematic review of over 1548 randomized controlled trials (RCTs) from 20042018 shows the promising use of UDCA as a therapeutic option for NASH. 